\title{
Report on the
}

\section{Twelfth International Workshop on the Identification of Transcribed Sequences: Functional, Expression and Evolutionary Analysis}

Held at Washington DC (USA)

October 26-28, 2002

Organized by

T. Wemer

S. Weissman

S.B. Hedges

K. Gardiner

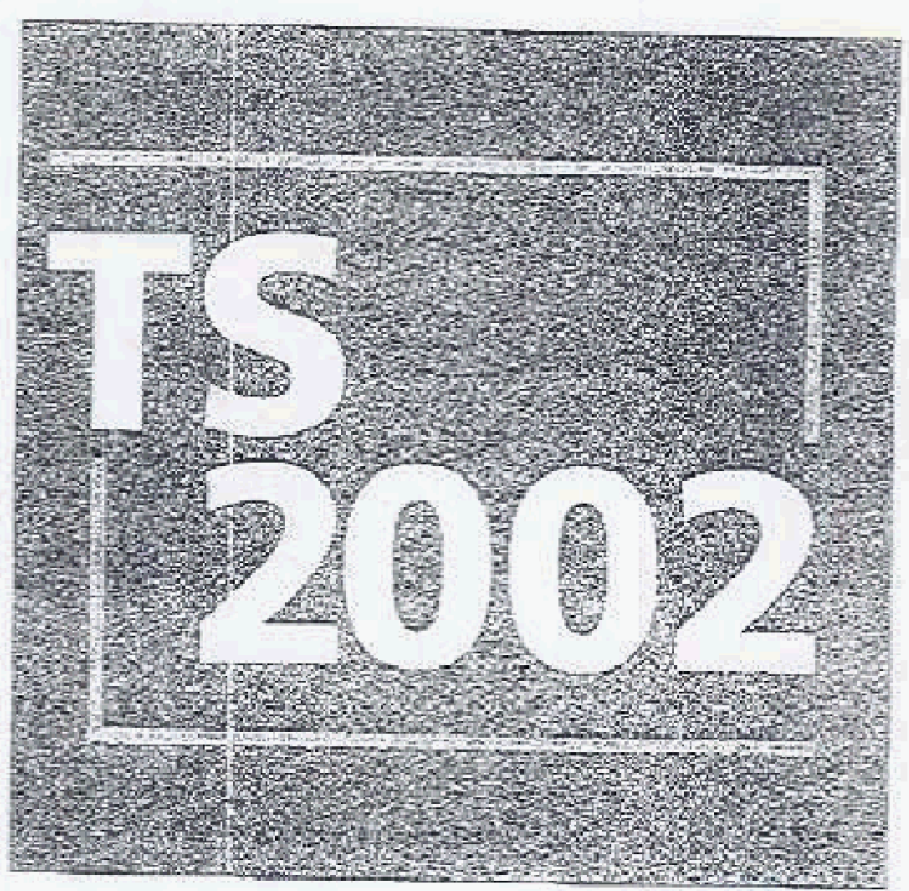

Accepted for buhlicacion: Decombar 8,2010 


\title{
Report on the twelfth international workshop on the identification of transcribed seqquences: functional, expression and evolutionary analysis
}

\author{
T. Werner, S. Weissman, S.B. Hedgest and K. Gardinerd

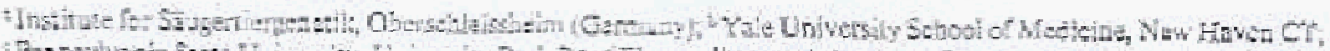

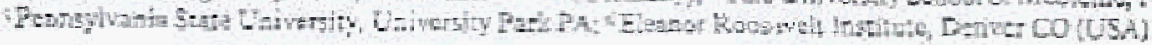

The twelnth workshop in the seris "Beyoud the Idertifiestion of Trangcribed Sequetces - Functiona!. Expression and Evelutionary Abalysis" was held October 26-28, 2002, in Wachingun DC and was again spontsored by the US Depertment of Enargy. The sevenay arrendes discussed a bread thange of topits retevant to genomies, gente identificaton. $\mathrm{R}, \mathrm{A}$ anshsis and post-genome-sequencing questions of functional awses-

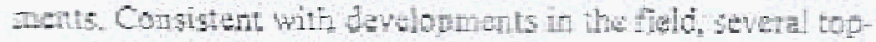
ies ractived incrased attantion and somb tow tophes were muroduced. In functional analyses, large-scale mutagenests projecs it rrouse and figh ate expanding rapidly, protein chips aro verging on prachicality azd deterninige qetwork conotctiong and interackions among zenes is being approached from multiple aveguts. In expression stuties, cDNt arrays reman popular but tow appleations are adduessing ud ditional un RWA characteristics, izeluding stability and tanslatian, detection of large scale basal transcription of apparedt intergatic and fmon sequences creates new queshons, and nonsenze mediated cecay provides potential thew insights finto functions of altemarive 5plice variants. Evolutionary topics included new sstimates of the timing of increased organismal complexily, pattems of grtomic and gene duplication in vertebrates, atd detection anong primates of buthan-specific soquences. Sunmaties of oral prostatations follow

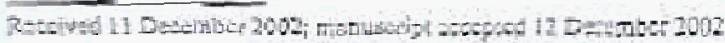

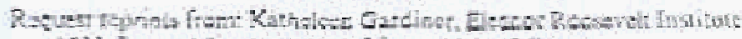

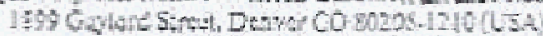

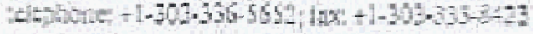
emil gardingratumenseds

\section{Mutation and SNP analysis}

Geerfray Hicks (Manitoba Institute of Coll Biology) discussed the procedure of fagged soqunce mutagencis tusieg retrontal insertions in ES cellg. Currenty $>4000$ lines with sitfo inserton ites anc avallable, with -33 h dianpting knowh gencs. It 15 ar ticlpated that 10,000 - 20,000 ES call himes boring ciscuitions of speific penes svantualy will be available. Data

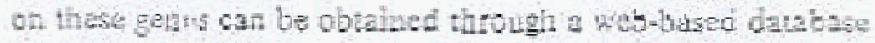

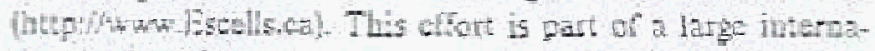

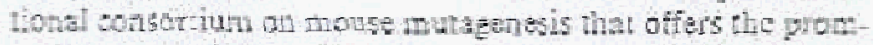
Ise in tive neal future of prowiding an ardy of motsc mutations Whit coveragt cornparable to the ichockout mutations avalabic tor the vest tenome.

Harald ven Mcleaner University of Frankfurt Medical School) discussed progress of tho Gernan Gene Trap Conss:tivm in exenting similit large-scalc mutagenesis of ES edIs Integation sites huve bera identified in over 7000 clones and i-cluda - 2500 known genes. Over 70 gern line chimeras have beor genefated with swer 60 of showing a phenotype, By using seweral genereap wetors it eppears that mest genes can be lar seted. Desa is beigo deporited in a publie database. This and the rhisted cforts discussed abveare noving forward at a very impressive rave cowurds saturation mutagenesis of the mouse Bensma with its consequent enlightenment at least for the sarlest escential unctions of mast mousc gertes.

Heabe de Angelis (GSF Center for linironment and Health, Neulugberg) discussel a massive effort to identily intersing dominat and restbive movse mutanis generated by BVI" mutagenesis. Currently, 405 anfinmed lines buve becn derepopd, of which 39 art recessive mutations aud 12 show belaviona phenolypes. This group has forused analysis in part on studisa of the noteh/ased sign alling system in wiek. 
Revenc Cindings inctude the knockout of a novel Deltal gene allet and its effects on development of the ondocrime pancress as uell as a role of the Noteh systen for inner etr dovelopment:

Adam Amsterdati (Massachusetis lnstitute of Technolos: Cambridge, MA) deschibed work from Wanw Hopkins grous on largosiale mutagencsis in tebranish. Their protocol tes nouse rutroviral vectors as insertionel nutagens end results it? Foundet fish with multiple insertion sitcs Sereal subsequst: crossts are required to genurate homozyaous mutants. To date. 550 mutations have been brad to homozygosity and come. sponding insertion sives has been doese for 410 of these. This bas iden tified 350 candidele genes, estimsted ro be $15-20$ th of all gente that wan be muiated no give a visible plienolype during the firs five days of zebrafis life

Complene SNF andysis for individual would cause a com. birmotigl explosion in data for exampla, all SNPs frotu just 1000 individuals would produce 20 billion possible phano types). Therefort, Sequenom (San Diego, Califomia) is concentraning on haploypus father than undividual SMPS and usirg DNA pooling to reduce the number of assays requirsd Michael shi descrbed how SNPS werc deteted by primer extension followed by MALDI TOF analysts. The integration of areas under peaks allows for a quavititative measure of the proportions of alleles in pooled samples, Common wariants ruay be associated whit diseages like diabotes, Alzhemer, HIV resistanes, or cardiorascular diseeses.

Sheman Weissman roviewed progess th the developmen: of utelhods using DWA glycosidases to roover nost or all mis Inatch-containg firagents from a poot of DhA duplexs. He furtio rowiewed the Yale Center for Evolutionary and Csconic Studies poliminay applisations or pertomic titug arrays to cetection of these mis.uchck-containing tudements and porentelly to wher 15 sues in tuammalian gunotrics, suth as global detection of Henscription Sachor binding sites (ChipChip) experiments.

\section{Protein analysis}

Thomas Joos ( VMI-Natural and Medical Sciences Instrtu, Tibinget revitwed the principles of protein microatray tah. totogy and disctssed applications including the parallel detetion of antibodies direered againzt a nurber of potertial zutoantigens. for detection and monitoriag tho progress of autoinmune disorders in men. This sype of array is only in the initial stages of development and application for bunedical purposes but offers similar promis. to that obtanged with eDNA micuarrays.

Michad Snyder (Yale Uniwersty) diswesed his eisgant work prepuring and analyaing aravs containing almost all pro.

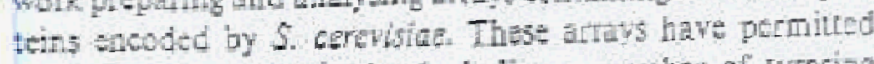
detertion of new actiwtics tucluding a number of Lyrosiae kmases. Other novel assays include detertion of proteins binding wo spasific phosphorlated forns of inosirol and diret: datection of probin-protcin tinteracuicus. Because he mammplien genome encods twwer than ten fold more ptoteins than dons yeast, these resulis offer bopt that in the future extemsive or nearly conplete arrys of manmphan protems might bscome arailabis:

Jeteny Sinuson (Europeat Molecuiar Elolocy Leboratcry. Heidelbers, Gomany) discussed use of VERO dells. very large fibrobiasts from nonkeys, for wisualization of protein localiz: fions in intratellular structsres of $1000 \mathrm{CDN}$ As targered for malysis, $>270$ have beec successluily localized to one or more of 12 comparments of the csli: with a furthet -70 failing to localiza che to intererence from the gneen Iuovescent proteir used as a tag. This system also allows direa monitonitg of pro tein iransport through use of a temperaturesensitive thutam of the VSVG pwtein, whth is blocked in the endoylasmit whou Lu at $35^{\circ} \mathrm{C}$. Lowering of the temparathe $1032^{\circ} \mathrm{C}$ releass the ER block and proteins that affet the WSV-Gproten manspre cett be dectmined by kaoktout studies.

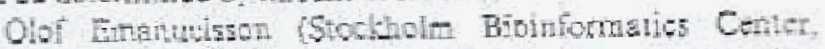

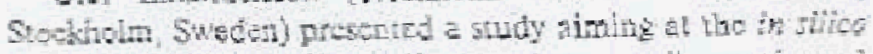
detection of proteins located in prroxisomes. Alt putoxisomal proteins cortaining the Proxisoma! Targeting Sigul! (PTS1) seguence (a tatal of 152 ) wers collected from Swisspot, from which a botal of 35 different PTS motifs were defined. Nonperowsomel proteins containing the PTS! signal were alse collected as a newtive control set. In this algorithm, the nine antno acids inuediately M-terminat 10 the FSTI were analyzod by a combination of neural netwoks and support vector machines, and the motif asceptot only if confimed by one of the 35 mown motifs. Further firesng by other predicuons (like Iar. golf) to remove othet subedlular compartaent specific proteins (e.e. transtwernbrane protsins) resulted in prediction of ocly 60 peroxiomai proteins in wigh hither eukaryoutc genomes with $80-90 \%$ of all maleing proteins contetly pre dieted ts peroxisonal proteins

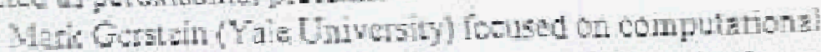

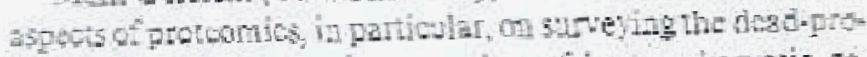
telu fossil pecudegenes in a number of harge cukeryoric go names. He icmifited presdogenes in yeast worm, fig and bumart. frodisu thousands ol now ones. Sonc of the friteresting fincings wase (1) The pop tation of presdogenes is not waitorn arongst protein familics and teods to favour envionmental rapotac genes (ii) There is a iange number of processed psetdogenes in the fumba genome mosty stemming from ribosomat protens. (u) The fy genone bas a wary small number of pseudogencs, checially compared to its cousin the porm. How: wen thig gan be explained through a procks of genome decey and one ear find hurerous broken up pseudo-motifs in this geisome.

\section{Large ecale conA analysis}

Ruth Weltuteuthe For the Gerwain Gancer Conter (DK- 2 ) in Heideltuty, Gerweny, presented the progress of the Gemar DNA consortim. They fosused on sizeselected RXAs for amplification and EDNA donith to avoid leogth bias. As of Oetsbor 2002 there were 160,105 dones avillable. representing 627 diferon genes, all of which ate boing cveiu. ated ha sertes of experimcrtal as well as in siloo assays. Also inciutud are functional protein ssats besed on protein chips 
and antibody drreys. The databaso is called LIFEob and :

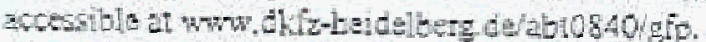

Takashi Gojobori (Natonal Institute of Gonetios, Ituan) described his research on the crolution of the central nervous systen. He and his group have sequanced nany cxpressed sequence tags (ESTs) from the brain of e pimitivanimal, the planaian (plaryeininth), and have conduotec microaray analyses. From this work they have identimed and character. ided genes such as now-daroke folk that play mporant rotes in brain davelopment:

Osamu Ohan (Kazuse DVA Reserch Instifute Riken, Japas) reported that a towal of $>2000$ KiAA eDNAs, reprosentig large cDNAs, and eveoding lerge (s i $00 \mathrm{kDz}$ ) proteins

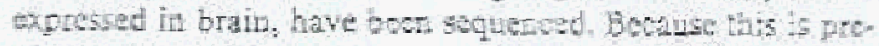
dicted to represent clope to a compleie set of such genes fitorts ere now bcing diccted towards isolution and sequemsing of orthoiogous mouse CDNAs, proparation of antibodies lo mouse protens, and analysis by yeas two-hybrid screening.

Thomas Gingeras (Aflymetrix, Santa Cland USA) showed data from non-repctilive sequence, genowio nudeotidt areys of bumal chromosomes 21 and 22 , scrused with cytoplanit cDNA from 11 cell lines (10 of which wera caneer efil lines). Surptisingly, $24 \%$ and $65 \%$ of positive probs were found in introt and incergebic regions (in at least five of the 11 cell lues testrd), respectivdy, wih a subset verilicd by RT PCR or Northern lybribation A number of regulatory factor binding sites (Fom e-ryc, $S P 1$ and $p 53$ ) were defined by chromatin immuroprecipitalios, within upsiream reions of the putatrve new transcripts, as weil as at the s" eqds of Wown getics. Thest daha siggest much higher trancerptional activily of $\mathrm{H}$ akiown function, than anticipated.

Merin Ringwald (The Jnckou Laberatory. Bar Harbof. Meinel reportcd on the ongoing developmant of the Mouse Expression Datalase (GXD). This doubase has now com. pleted hiterature analyst for gome expression duine the mouse embryonal development front the ruar of 1993 to present. Ringtale also proposed to wse ormogond vocelularies to describe phenoment, such as functional tenas (e.g. incuce of causc) together with anatomical temm. (e.3. bones o [ivet) And medicel uens (es. cancer or diabersis). This results in in alnogt tatural languge description that abides stictly by the rules of eontrolled vocabularies and is thus cortputer processible.

\section{CDNA arrays}

Dr. Yoa' Arava (Stanford Urivenity) studted inanitational fficieloy in 5 . cerevisiad by expenments measuring the disirbutien of $n R$ NAs assowated with golysomas. This was coupled wh a method for speific cledrye in the centril ponion af an MRNA. Overall conclusions werc that elongation is processive atd thet temiration is not a slow zep in protein generation. bus that mitiation is fate liniting for muat mRWAs and, in gen. erd, longer mRNAs, ft particular thoge with long 3 UTRS, are iransated less effeisnty beceuse of alowe initiation 1 atd.

Frouths encoding RVA Reognition Motifs (RRMs) rephw sent the sevcrth most abundant protein farily and art likely to play divers toles in reguation of many features of RWA procossing and metabolism. Soon Tenenbau (Duke University. Madieal School) raported on efforts to correlate individua RVA bindeg proteins with theit target mRNiss. ProwinMRVA conpleses were presipitated using an antiboty to the speotic RVA bindine protein (e.A. FABP, FNRP), and ascociatod RWAs are then labelled and used to serenn a Clontoch CDNA arrat Unique subsens of RNAs have betn identimed. allowing further analysis of reognition seruenees.

Aravind Raghavan (Lawarsity of Minncsota) discusse analysis of HRWA decuy rates, using actromycin D to preven: now nRNA surthesie, in I cels before and after activation of the exh. He obemed thany short Lved transcripls and inporianly, ran transeripts thet ware enber atabilized or dostablszed by cell activation. Many af the transceipts rostated at the lovel of nRW A sublity ponteined no previously chatactersed stabuly documanus, cmphasizing how much remaite to bo levrued in th is ficid.

Dr. Michael Licbiet (Raytert GmbH, Straubeihard, Germany) prasented data on the warjability of ragults at arrey expormears cansed by different sotware evaluaion stategies tor spot detewion and background suburation. Coupharison of three spot detection unchods (variable spot arba witl atocon. touring of spot shape varable spot dianeter and background subtraction, anc constant spot dianuter and backeround subtuaction) sbowed no significant differences for spots with high signal intens stos. Howere, for low intensily signals, the spor detection wh varible spot arce with autocontourng of the spot \$hape, as well as the variable spot dianeter demongtrated a ciear tendency to undencrimste or ignore spo: data.

Michal lither Wationd Institute Dor Genome Rescard. Buthesta. MD) described insightul interprotation of CDNA

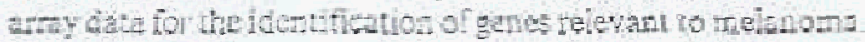

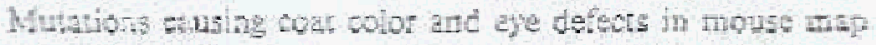
near the Rabld gene, implicaced as a candidato gene for hunin melaกที

\section{Expression notworik:}

Thomas Werner (Gevomatix Software GmbH, Manich, Cormsny prusented studies with the PAXs gone as an shample. of in silfo delemination of potential targeis for transcription facos (TF). For most pronoters, muitipla TF binding sites are requifed and trascriptional response is only ehcited by two or. more factors trinding simultarevusly. For $\mathrm{P}$ AX3. this computa. tonal approach helped to reduee the number of candidate ta:get genes - 100-fold leading to the identifeation of a fuctional PAXS notuork in signal tranduction.

Sorin Istinl (Celere Genomes) deseribed the Davesurt model of trancription regthation foveloped wo son unthin. and formssine on the endo.6 gene tand its assobitad network pif Itemthons. Trunscripion factor binding sites and pomoter

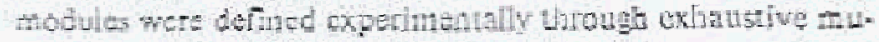
tational ansysis. Mathematioal nodels of the neqwork and

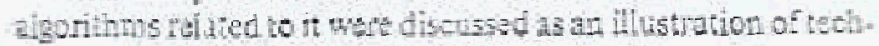

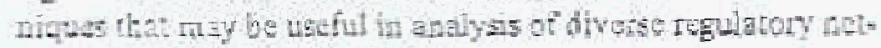
warks in this and oldaz organisms. 
Katheleen Gardine: (Blcanor Rooreveil Institute, Detwer, co) desenibed how perturbations of protetn netwarks and interactions, rather than single chromosome 31-gene overtx. presgion, must be the basis of the mental retardation phenotype of DowI syndrome (DS). Among cxamples diseused were sevton genes rappline to chromesone 21 that directly function in pre-mRNA splicing or the spliceosome, syggering a global disruprion of alternative splicing may be cuatributing to the DS phenolype. A second example involves styer gores that intsFes: with components of the MAP Kinase pathway. Preliminary deta suagest that the MAPK pathway, in particular, leve's of piresphorylation of ERK1/2 and Akn $1 / 2$, alestered in braims of the mouse thocel of DS.

Alis Brazma (European Bioinfomatics Institure Hinxten Cembridsa UK describod ex spproch to deduce gate ne!-

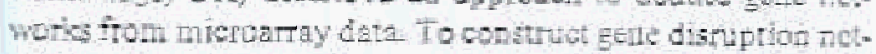
Work, the micro anry date are firs discretized into $t_{2}-$ and 0 , by using suiable filters. The telative distribution of itcoming and cutgoing adiges across a complete set of genas allow's con. strution of sede-iree nerwork. Genes associated with a high dagree of ourgoing odges are reguatory genes, while high degres of inzoing edges usually are menbolic genes, Ramoving ore node at a tine creates independent sets of genez connected only to cach other, within sets that are called network modults:

Suchir Kumar (Arizona State Liniversity) reported on a sew computational approach for comparing gene expression patterns from early developmenal stages of Drosophile meleno gaster. A ducabnse of expression pattems was sceated through digitization of avallable publisied inages (thousands) and computatioral wols were developed to seareh the database using qusy inages, analogous to the rav BLAST Find similar seguences Such methodolagy eould be exteaded to othet orgenisms aud should scceleratc discovely of dewelogmental metworls.

Turgen Bode (Garman Biotchnology Institute, Bramsthweig Germany) reported on the use of a biomathematical method callet Stress induced Destabilization of Doublustrands (SIDD) to sutessfilly predict functions Scafteld s: Matrix Antachmant Regions (SMARg) in the Interfeton type I gene dusicr on the short am of chromosome 9. SMARs predieted gear the $\xi^{\prime}$ ends of guncs sould be confirined by single-stand analysis, while these SIDD predictions locutcd within the cot: ing region showed no SMAR activity. DNA mechylation aFpeared not to be involved while secondary strueturs potential is an irmportant SMAR feature.

Possibly relatcd observations were described by Alla Rynditch (Instrute of Molecular Biology and Genetics, Kiev, Ucaitel, who has shown that the algha-giodin genes in chicken lenkemia culls are transcribed and stabilizod as a singta "tul] dowsin transeript" spanding $30 \mathrm{~kb}$ and containing all three apha-type glovin genes. This large transcip: exists in spue of the preserce of interual CDG istands and SMAR Interescingly. it is found as a component of the nutlear matix and unns over slowly, possibly sugessting a novel mectanism of post-trar. scriptioal feculation becauge thess thobin genes do not uppear as proteins in these cells.

\section{Alternative splicing and pre-maNA próessing}

Lybue Macuat (Univesity of Rohester Medical Center. Rochester, MY) discussed aspects of Monsense Modiated Decay and its raiationship to alternative splieing events that introcus: premaiture stop cooves Stefan Stamu (Insticute of Biochemiswy, Etangen, Germany) discussed the role of phosphorylation in aternativs splicing. Specific exanples inelude the $\mathrm{CDC}$. like inass5, Cl.K1-4, that phosphorylatc serme-arginine-rich proteins, that in turn regulate attonstive splieing, abl-rudiated phosphongtato of SLM2 which results in cxon skipping in the tu proteir mR $\mathrm{ma}$, and insulin treatment altering phosphorytation of SRpto which it sum ahers atemative spltcing of PKG.

Winston Hide (South Airican Natiosal Bientormatiss Institure. Cape Town, South Africa) analyzed RatSeq and IbEST sequences to dustribe blternative splicine events caused by exon skipping. Because $90 \%$ of such events oscur in soding acgions, they affeer protein eoding, with $-50 \%$ resulting it rading fume truncstion. Currety, the observed fiequerey of cron slipping in mouse transcripts is lower then in buman, which nay simply be dus to the lower quality of the mouse genomic assemily, To correlate exon skipping events with disease (s.g cancec). Hide proposed a controlled vocabulary to dascribe ontologies for cell type, developmental stage ane patbology, thus permitring theit lishage wo mascript acoession jumbers,

Kirk Jengen (Rockefeller Lniversity, New York) also reported on efforls to identify tarzets of the RNA binding proteins, MOVA and Hu, relevant to nervaus systeth developaren. A rowet protocul, GLIT, has been developed in which RNAprotein eomples as are photo-arcsilinkef in sin directly in net. Thts. Ihe RNA is ther elipps to the 36-60 nucleotidas pro: retted by the protein, followsd by fmmune precipitation with sppropriate antibody, and putfication dud eloning of the RWAs Analysis of 130 RVAs ins shown the following dretributivn: 75 \% are lowalec wituin introns, 20 \% are adjacent to altarnativly spliced exons, $20 \%$ are whin 3 UTRs and $2 \%$ are located within soding cxons. These data suggest potennially divere functions retevant to provessine and stabilicy-

The ADARI protein functions in the deamination of spocifie acenosinte residues in spccific pro-mRNAs. Although wellchareterized substates include serotonin and glutamate recoptora, Jimy-Hua Yane (Yale Univarity) has shown thrt ADAR tastivity is broader than that. ADAR1 aetivity, as monitorod by tnosine (the direct produst of deamination) produsuon, is increased in spletn, thyms and lung during infiammaLion. RMA and proten isoinm petherns are also altered, mos: oten afierting the nuclese localization signal, the Z-DNA bind. ing domain or ane of the RNA binding domains of the protein. There novel isolorms gppear to be responstble for s shift is boulization frota the nualeus to the nueleolua and the wytoptasm, sugasting pussible novel functions for ADARI 


\section{Cene organizational analyses}

Iee Rowen (Institute for Systems Biology, Seatle) describsd the identification and organzation of 211 human genes each spanning $>500 \mathrm{~kb}$ in genomic DN A and thus together accoun: ing for $-5 \%$ of the Kuman genone. Such genes tend to be located within. A T-Iich regions, exhibit highly complex alter. tive splicing, alihagh wature mRNAs averagc only 2.9 kb. play aritical roles in developmentand physiology of the cestral pervus system, and are fruquently locsted at sites of chromot somal instabitty. Lasty, the latge sizes appear to bie conserved if mouse.

Yoran Groner (The Waizmarn Ingtitute Rehovet, Istule? distussed the fandy of three manmalian Rumx transeriptiont faeto:s. Rurxl (nee AMLI) is ofter distupted by chromosond cranslocations in acute lelkemin anc is essential for denntive hematopolesis. The gete is trinstibed from ather of wo widely separated promoters, one of which produces o s urrenglated region of over 400 bases and includes an IRES. Runk3 is also transcribed from two sltmative promoters. However. unlike Rupxi, kuockout of Runx 3 leads to a specifie neurolog:pal deficit (sevcre ataxia) as a consequence of apoptosis of a subsct of dorsai ropt ganglion neurons.

Tom Blumenthal (Univensity of Colorado Health Seiences Center, Denver, CO) deseribed the identifieation and tharactenzation or operons in $C$ clegons, where a total of 2327 genes ate found to be transcribed as 898 operous. Frequently inelveed in eparons are mitechondrial genes, and genes with roles in thaseription tnd araslation; excluded from operons are transoription factors anc sollanes genss. Inturosting addy Wonal foutures of operons are that they are not defined by thetr promoters lswitehing an opan prometer for a nes-opatos promoter still results in Lanscriotion as an operon), asmponed genes do recesuilly appenr in equai levels, and componerit gencs are not nesessarily obvousiy functionally ralated. Lastiy. What operon components are functionally related, they nay providie a usefut toul for analysis of or hologous human diseage related genes.

\section{Evolutionary and computational approaches}

Xun Gu (fow State Uniweraty) diseussed his recent wor: testing two tbones for the evolution of the vortebrere senome. Doe model suggests tha: there wene two compicte groume dupitications (polyploidizations) in the history of vertebretes

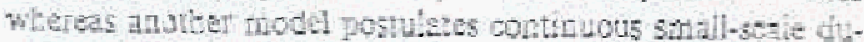
plications. Fis dating of 1.739 gone duplication evene perided suppoit for inciusion of both motels. in wolwitg ar teast ota tomplate genone dugteation followed by i wave of small. a-soele duplications.

Blai- Hodpes (Pennsywant State Luiversiy) dxelussed some tecenl bioiniormatic studies in his laborutory conecrning the crolution of conplex multedluat life. The tirses of divergence belsewn selected protists, plants, avinals, and fung wer cstimated uine seguences from 50-iso gones each. The results shorw the: organismal complenty inereased much earligr (1.5 bilion years ago) than previously bolieved and about a bitlion years bufore the Cambrian explosion of animats.

Naruya Seirou Gational Institure of Gematics, Japany reported on work from his grovp, with the goll of adeatifying genatic changes terponsible for mocphotogeal traits uniçus to hun:ans: As a pilos projot, they rargetro the HOXA gese cluste in humans and apes and seguened 21 kilobases (100 tilobuses for gotillas and thmparzes). including ine HOXAs, HOXAS. HOXA6, and HOXA7 gens, whin hise data they were abie to identify several hor DNA regions of potential valuc in zodressing these evolutionary questions.

\section{Roster of Participants}

\author{
Adem Ans:erdan \\ MIT Cenler for Cances Rugench \\ Mr \\ 77 Menchusetts Avenue E17-340 \\ Cunbricte, MA 02138 USA \\ Telephon: 517.2539009 \\ Fax $627-258-0238$ \\ Enafl tiogentited \\ Yave Arat \\ Biveleatisty \\ Branlort Medical Behoo. \\ B435 Bstionan Centus \\ Pulo Alto, GA 94505 USA \\ Telettones 650729-6719 \\ Foc 650725.6044

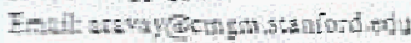

\author{
IJas Bereste \\ Deparuent of Hunbat Gesciss \\ Wellkcre Taust Senges Instutu \\ Hinnten Gubridge CBIC ISt \\ trited Kirgdom \\ 7etphone: $441229-494732$ \\ Far: $44-1223484919$ \\ Enail ipegungen: \\ Stephanis Jleothd

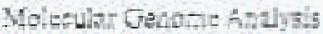 \\ Geman Cathe Recereh Canto \\ In Ncuphitumer FoC 280 \\ Heideiber. D-69120 Cuman:

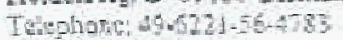

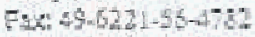 \\ Enallsowhtegekiz os
}

\author{
Mighe: Bittoet \\ NHWHGRI \\ s0 south Drive, Bidg 50, R n 5154 \\ 3ethesta MD 2082 USA \\ Telphone $301.496-7980$ \\ Fax $301-402-3141$ \\ ETail mbimmengatgriningow \\ Therarinn Bhodut \\ Wew Toltuthes \\ deCODE Gendies, Itic \\ Srutilugala : \\ Reyiterath, 101 lseland \\ Tesepone $354-570-1379$ \\ fxr $\$ 54570-1909$

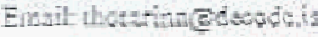




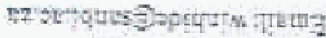

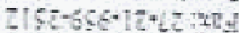

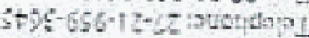

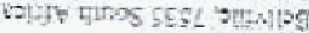

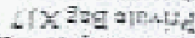

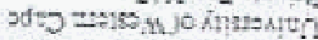

oh1

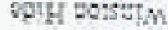

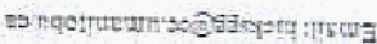
o6t-ik6 $\rightarrow 02$ MI

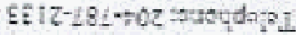

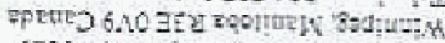
650s No my'

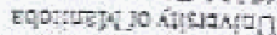

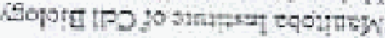
EMTH yos

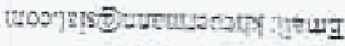
ct9l-98ctir:Me: $9653-892+15$ oudpata

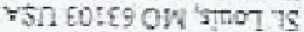

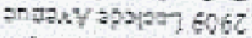

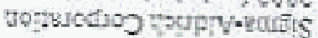
말 atcuxandf toy

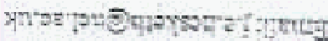

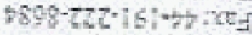

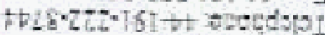

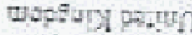

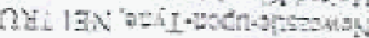
provgrus:

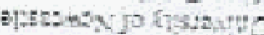

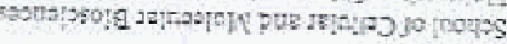

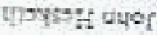

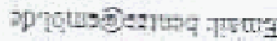

$81648: 1209+6=503$

$105486-1725-5 \%$ anomdepl Aremiso 41 I69-q hearspla

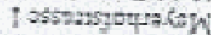

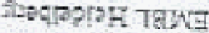

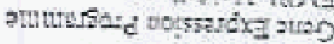

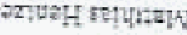

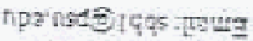

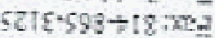

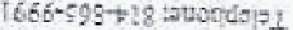

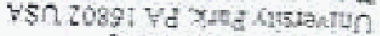

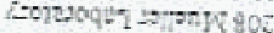

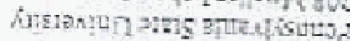
Hopres nothutbo

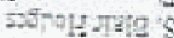

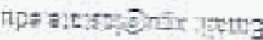

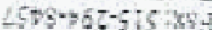

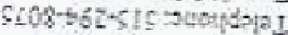

USר IISt vT thar II Homestos tes

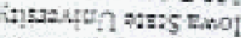

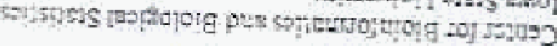
$\operatorname{moth}$

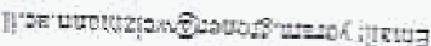

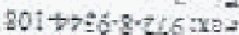

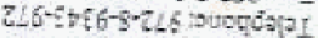

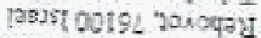

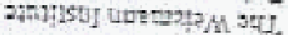

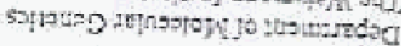

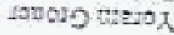

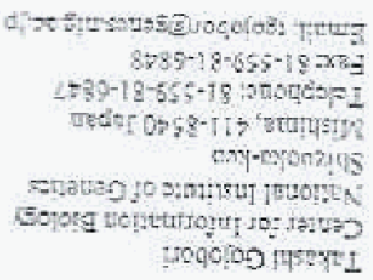

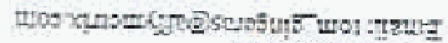

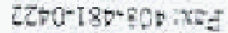

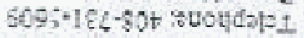

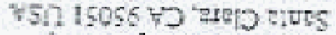

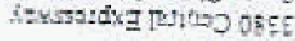

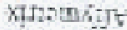
dnarg surat:ass-4I

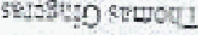

n) v. $5415-20+-\cos \omega t=$

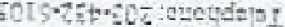

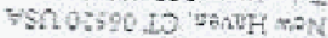

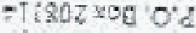

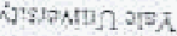

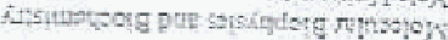
HFon Xite

mptrats â,

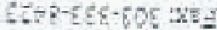

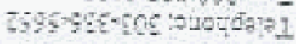

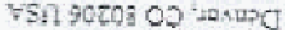
thes pueteo ESS:

क्षमा: thup reg जrejapus

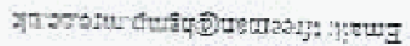

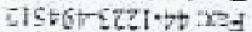

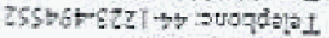
"

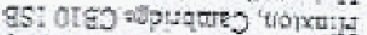

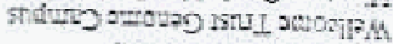

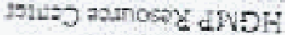

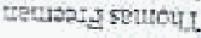

450

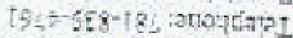

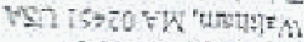

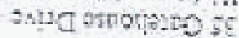
wantzonts जबास्दा वश् Hมพ-

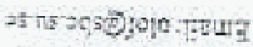
$7+28+255-8-9 t i n c t$

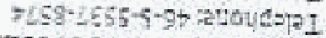

uspang $16901-5$ 'Worroons

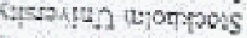

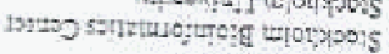

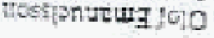

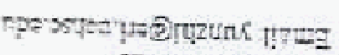

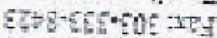

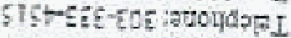

Wan 50108 og ventact 1275s propiso 668?

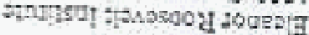
na $\operatorname{trntan}$

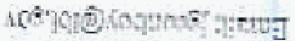

$7+49-58.015 \times \mathrm{xg}$

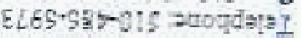

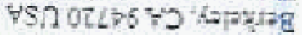
paoy ualowis I

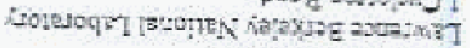
wortaic rowes ant Koqिएक सror

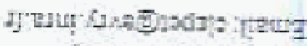

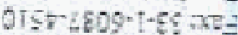

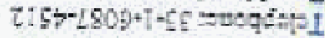

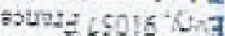

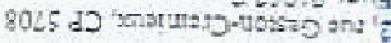

SAlv Move

NIIS UEMTHS

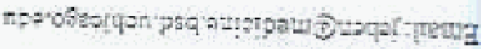
COOE-60L

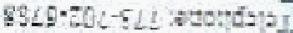

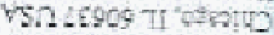

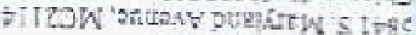
DEvTh I0 hrgat: cago unflug:?

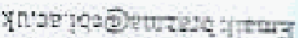

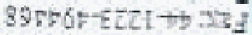

$\left.89,5,-4 \mathrm{~L}^{\prime \prime}+2=20 \mathrm{~d}\right) \mathrm{s}$ wod:us pepun

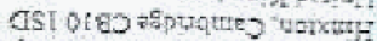

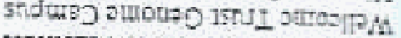

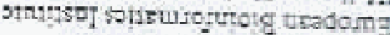

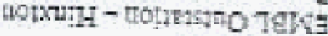
EUraकe 5:

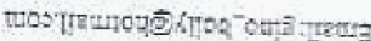
$46-5+5 t \rightarrow 15$ ars

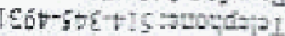
Eprtho kot I

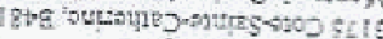

Fifdsor pungm rembs

Mgont-stary

Âtge crs

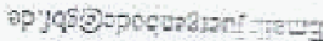

$29 \tau^{-18}-19+195-6+x=3$

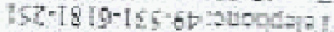

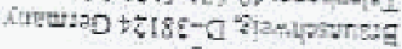

1 ks.4 tepanoung

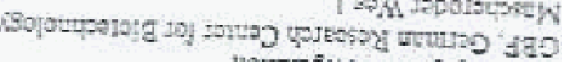

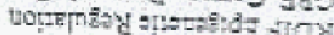
spog coltur.

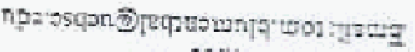

$5170-515-60 e$ ket

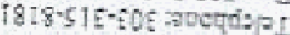

TSA 2508 os 5ama

Tzl-g Xor anns.

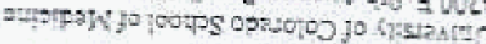

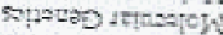

ptr Aryus pora ye istmadoc

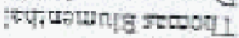


Naney Hoplens

Mir Center for Caneer Recurc:

MII

7T Massebugen a venue, E17-396

Cumbridge WA U2138 USA

Tilephene 617.253-6녀:4

Fax $617-2530258$

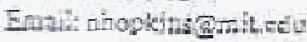

Marin Wrabe cangelis

GSNatena: Reserth Cente fos

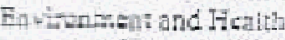

Inthute of Extrimanal Gentots

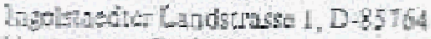

Xeuterberg, D-35764 Germany

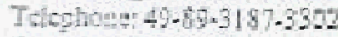

Fax $45-89-9187-350$

Fndit: hrabegut do

Emen Lteril

Infomatics Racoareb

Chicra Gerionies:

45 Wet Oude Drite

Rocking, MD 20890 USA

Te:phvitu 240-453-3668

Fa: 240-153-1324

Gnuir sorit istmilgetere,con

Stinise ivashita

Mitsubih Kaghen tostitute of Lifs Stioass

Minsmigoya 11 . Mathide,

Toko, 194-8S1L Jquer.

Tolophope: $31-42-124-6265$

Fax: $81-41-724-5316$

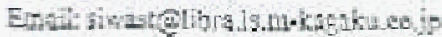

Letejeftory

De- sopmentel and Reproductive Biskg

Biogedicum Tekins

Hourtmanik katu 3

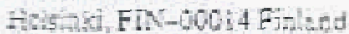

Folsphoue 5:8-1912-5652

Fit $35 \pi-5 \cdot 1912.5684$

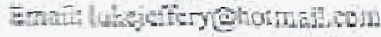

Kirli Jaese

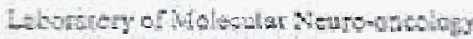

TEt Rackethle-Tuvesily

1250 Yoekswenu, Bax 226

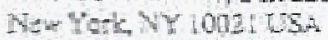

Telephone: $212 \cdot 327.747$ !

Fex 212.197.7109

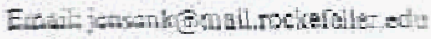

Thow as Joes

Thetiral and Medied Scionocs Institure University ou Thingen

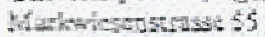

Fcutingen, D-72770 Gentlan

Trisphste-49-7121-51535-544

Fer $40-721-52530-16$

Eutait posennide

Jater Ktotet

Molethe Gunches and Microbiojogy

Dufe Uainexity Medieal Cante

4le Jumas Buldine Resenda Dive

Duthan, Ne 27710 UBA

Toleztroe 915-694-5138

Pux:9194048735

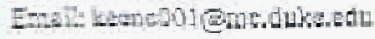

Arit kalt

Molecular Gonome Andyis

DKF 2

Im Netahnguner Feld 2b:

Hedidter. D-69:20 Comany

TCiephone $49-621-4247$ t?

Faxi $45+6221-423454$

Emsil: a tolbQdico

Bemasto Kom

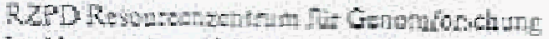

In Newblbines Fold sos

Hedelexm 3-69l21 Gymany

Tolsphore, 49-6121-42-4700

Fax: 45-512:-49.4704

Ensi: korngeds:

Suphon R̈ruste

MCEldOBGM

Waphe 3ture Uateraty

275E Hanco 253 C 5 Mon Cuth

Detron, MI ABat LSA

Tulephone: $313-57-60770$

Fix: $313-577-8554$

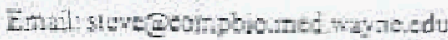

Suthir Kuns:

Genter for Evolotionary and Funclienel Gi somics Arizons Sute Univerity

Life SGences Ais:

Tumpe, AZ 8325\% USA

Teipeliene 480.727-604?

Fax: $450-585.251 \%$

E-ail sumigrcosused-

Robart 1estimman

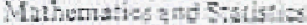

Merand siatu Lnivisty

73625 Kathine Drive

Tempe, AL 85253 US:

Taichone 40456956

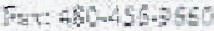

Endileramangogitheo=

Mtions: ticbles

Rayles Cats

Ranaiscusso 15:-

Sichtzl, D-7263. Oumpar

Telephous 4 a-7082-925:0

Fex 49-703-208:3

Emailh habererates de

Cani-YoLin

Gancine Irsilute of Sincepote

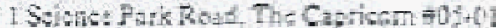

Siagapord, 17528 Siltgapor:

Foluphone: 65-6527-5210

Far $55 \cdot 6827+32104$

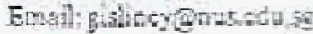

Guristian Maetcle

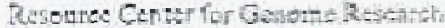

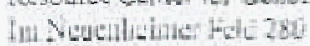

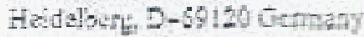

Talephose 49-6:21-24741

Fas $49622:-7254 \times 0$

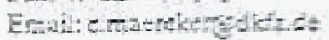

Iynge Mana:

Deparandi" of Bichtutustry and Bigahysico Uliversity of Rochester

G02 Elnwood Artnue, Box $\$ 12$

Rocherder, NY 14642 USA

Telephous 585-273 5640

Fax: 586-271-2683

Enail lyno-maque eurmatodieste-edy

Curoline Meduebs

Blentbonocolog:

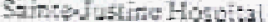

3175 Cois-Saine Cahuring. BCs!

Nonirah Queter H3T ICS Candit

Telaphone $314-345-4931$

Fax:51454-573:

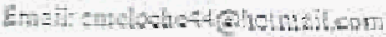

Dental horgh

Departecut or Cleststy

US Merat Aleadem

Mht Filloway Roded

Annpolit MD ZTEO2 USA

Telephos $410-29+6012$

Pex $410-292-22.8$

Emuit: monetelsandu

Onatu- Chars

Kozure DNA Rosegreh institute

2.6-7 Kogus:-Kamats?

Kinnar, 292-0818 Japan

Telohone 31-418-52-3913

Fox $81-422-52-3914$

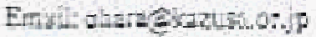

Amind Raglorin

Deperinst of Mterobisich

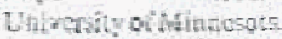

420 Dolawire Sno: SE MMC 196

Minncapols, MN S5HSESA

Telehor $\$ 12-6240462$

For $61502=0723$

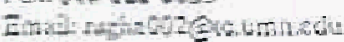

Fiandigen Re:

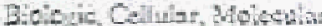

Lastitut Muscipal loursligeio Medies

D. Aisuader 80 .

Butsiona, 0300; spain

Telephore: $24,13-225-756$

Fax: 34-5j-221-3237

Enzit: pralginimict

Marta Riabueld

The Itocon Latura:05y

SWMate Suree!

Ber Harbo M K0460y USh

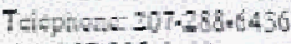

F.x:207-285-6131

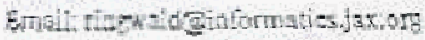

Lef Nosect

fistinte fur gysicins bulupy

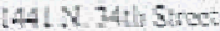

Seatle, WA 9810j USA

Teleghar-205-732-1372

Fox 205-122-1299

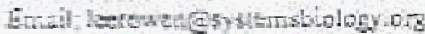


Aili Rugefielt

Ifstitis of Molesular Biolegy and Gsantes 150 Zabeictugo Sureet

$\mathrm{Kiev}_{\text {OB }}$ 1430kesint

Telspatos:39044-266-34-9.8

Fate 38-044-25E-07-59

Emant nadithetmbgargut

\section{Naruye Saltou}

Dlvisiog of Pogulativn Genztiss

Merienal Iestitures or Gunctios

Kisbind, $411-95 \div 0$ Janar

Tulephene $81.559 .81+6750$

Fax 11-5s9-81-6739

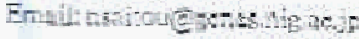

Sthestubudtan Sakargubrarania

Depontatit oi Biolces

Arroge State Universisy

Lirc Stiantes 324

Intupe $A 285287$ USA

Tclephog= 410-965-209]

Fu: 480.955.2519

Eimelit sampasuedu

Marite Becrem

\section{Genats}

Lowexity of Pennyluasia Mether Sthed

4.5 Curie Blvd, 560 GRE

Priladtiphes, PA 19104 USA

Telephane $713+74-6972$

Fite $215-5735992$

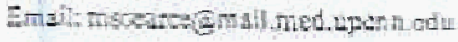

Mintuet st

Sequensm, link

3395 Johe Hopkis Court

Sun Diro Ca Bt:21 usa

Thisphote 350-202-9122

Fax $555 \cdot 2019127$

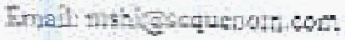

Jeneray Stmpoen

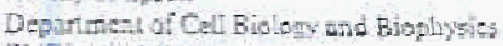

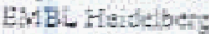

Mit, trbotsuse i

Holdelbet, D-69:7 Gemany

Telephont $40.6271-337-232$

Fex:45-621+387.300

Enat: simpochembl-heide bordod
Miel-ael Snyder

Molocular, Cciluar and Dewa"tpmontri Siologn

Yute Unwersity

R.C. Box 208102

New Heven, CT 05520 USA.

Tellophone: 203-432-6152

Fax: 203442.35:

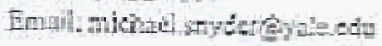

Safan Stam

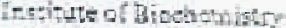

Linivesty of Erlangen-Murmours

Falratitis: 17

Extanger D-91054 Cuman:

Teiephus $45-913-142462$

Fax $40+2121862+4005$

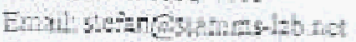

Euganswatem

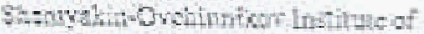

Bico panic Chetistry

Rusian Andorny or sejtencs

$16 / 10$ Milituldo-Matieve Sirnat

Moscow, 117997 Rusile

Trleptone: 7.95.330-652

Fax: 7-95-330-65:5

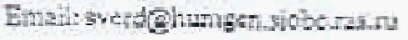

Soom Tenenbarat

Centher fo: RWA Biology

Duke Universicy Wecieal Center

406 Jones Blvd, Box $\$ 020$

Duthan, NC27 10USA

Thistitent $919-6842714$

Fax 919.584 .8736

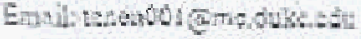

Hath ton Methiner

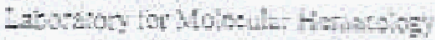

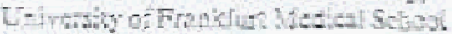

Thedot-\$min:Ri:

Fanhur am Naln, D-60590 Camasy

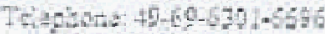

Fax $45-65-6301-6390$

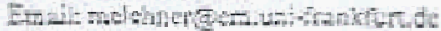

Malswim won Sehing

Biomediel asd Dife Seichers

University of Surrey

Guitord, Surrey GUZ 7KH Uniod Kingdem

Téreplons: $441433.686-468$

Far, 5013213-715?

Enall mon schanzgurrey ac uk

Shernun Waicerng

Ta: Uaiverity Schod of Matibing

298 Congress Awtus, Rm 336 BCMM

Nete Haven, CI O65lo USA

Telcotone: 203-737-2281

Fax 201737-22st

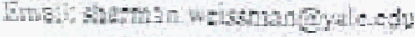

Ruth welenratber

Melcesar Gecoter Anulyzis

Gornes Canoer Reasardi Genter

17. Rewenhiner Feld 280

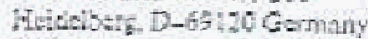

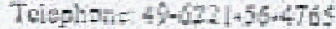

Fax 49.5221 .56472

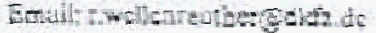

Thohas Wieries

Instive of Expenmentw Geretics

Nalional Rescurch Cente for

Envionmentand Heth

Ingolstadter Ludsentse:

Neuherberz D-S57gt Germany

Teichone $45 \cdot 59-3137+460$

Fori 49.89.2187-4400

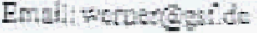

Jieg Fius Yas

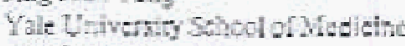

295 Congess Avate

New Hare-CTOGSHOSA

Teicphona $202737.55 \%$

Faㄴ:20\%-797-1504

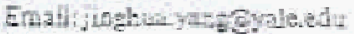

Dorethea Zin:

Moksular Genenge Anslyws

Gertuan Gatike Reswatch Center

Int Nouraheiner Fold 506

Heidebars D-69nio Oemuny

Telephore $496211-42-4712$

Fex $494221 \cdot 42-4704$

Einail d aink ediszds 


\title{
Report on the thirteenth international workshop on the identification and functional, evolutionary and expression analysis of transcribed sequences: comparative and functional genomics workshop
}

\author{
A. Brazma, ${ }^{\circ}$ T. Freeman, ${ }^{\circ}$ K. Gardiner, ${ }^{\circ}$ S. Weissman, ${ }^{\circ}$ T. Werner, ${ }^{d}$ and \\ B. Korn'

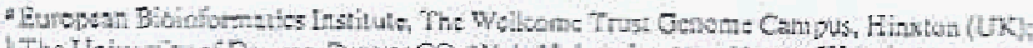

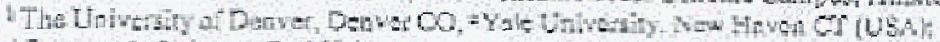

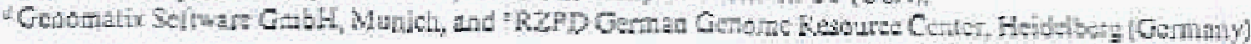

This thireesth workshop in theseries "Beyond the identifi. ation of Transeribed Sequenes - Functonel, Exprcsion and Evolutionary Analysis was renamed the Comparative end Functional Gemomics Workshop and held at he Sanger Center. Hisstan, England, Navember 2-5, 2003. The meting was 5ponsored again by the United States Depurmen: of Energy and for the frot time slso by the wellcone Irust fhe sighty artetidees discussed a broed rapge of topios rcicrum to geno structure, and functional and raguiation bnalysia it the era of compite and traft genomic sequascas from muliple organ. itits.

\section{Arrays and network analysis}

Mieheel Sryder (Yaile Univcrity) discussed the status and utility af arrays of yeast proteins with applications to the identification of kimase substrates, interacting proteins, and proteins binding to specific phosptorylated Foras of inositol, and alsc review ed the use of arrays to demonstrse antibody speciateity or the lack thereof. These exampits showed that pustetn array

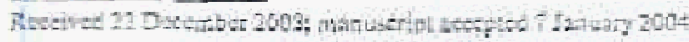

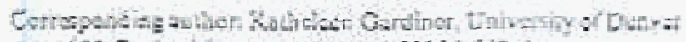

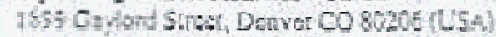

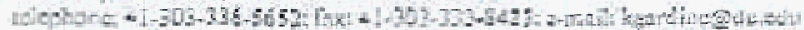

provide a praet ieal approech to obtain data in e veruety of bischemical situations, ineluding thos othawise requiring lebofious oneby out bochenical analysis. Nek Luscombe (trom the Gersccio leboratory, Yale Universicy) pooled a variely of Eata front che literature conrerring yeast protein-protein intersetions and genc so-exprassion studtes. While the initial grapt of all intcration a gpoard uninergtctably clonse, use of generIi rework parimeters and ifentification of specific aetwork "motils" helped to dissect out rasponses to speciffe stimuli, and tapid, relatively codined, responses could be distingushed from more conple interactiwe ones. This clear presputation olferct the prospect of evenual interpretation of yche intersefion parterus from compler, diverse and extensive data scts.

Sheman Wissmen Cale Lniversicy revicwed mRNA ckpression pattens in various lineages of the hernatopoietic 5y5tem, emphasiziug that etch lincage was characterized by a hight ly distintive quanticative pattern oi gent expression thal, notsbly. includes even difforances it expression lavels for thousekeeping" genss. This wark included deseription of a coll lin: that conthuously propagated two different forms of hematogoletie prosirsu cells as well as throwing off more differeh-

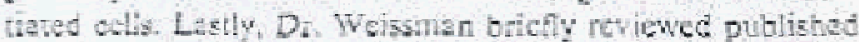

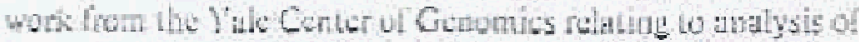
Lanseribed sequenes using genomic tiling arrays for chromo. sone 22 and of transeription factor batuding within chromosome 12 as anaivzed by dromatin inmugopretipitation plus genomic ning array hybrizization. Appreximately talf of all uenseripts in placenta derived from tegions of the genomie

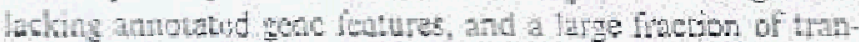
soription factor bincing sitcs ocewrred clsewhere than in the

\begin{tabular}{|c|c|c|}
\hline KARGER & 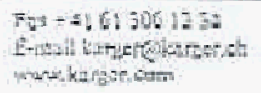 & 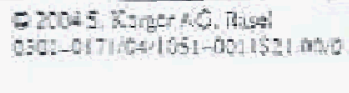 \\
\hline
\end{tabular}

Redumin ectipast: tew.enctast cys 
proatobers ol known genes, othe in fragnents that did not thave rocogniable binding sites for the Factor in cuestion. In sirmiar expechnuts wih genonic tiing furays and Lanstiption lactor bindige oxperiments, D. Oinguras (Alfymerrix) dessribad progres in preparing sets of aligonucicolide arrays thing tonomil sequances at densines of up to onc oligonurlestia every tive Dasez, providing demarkatic resolutior. partioularly for mapping sitcs of transestoed sequencos. An imprcssive number of these "novel trenseribed sequenes have almaty been cloner by RACE methodology, anc their furthe: analysis is awaiced with gneat intwrest. Together. these deta show tbe need for add. tional teanseribod sequence identifeation and vorification profects, as woll as hovel incromentions

\section{Regulation af gene expression}

A series of talks rocused on apecit of gene rogulation, Jang tra from direct identifestion of promoters aud chromosontl domains to plysical elustering of funclionaly rolated genes and adapked evolutionay ratss of highy oxpressed ghnes. Retrowi. ruses, sapecially HIV that integrate inte the host gonome obviqusty are also subjecl to such forces and segn to achot to ceilular anceluanisns.

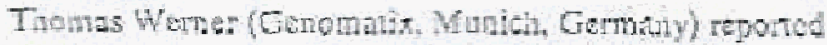
on recnt developments in the EIDorado systcm. which now includes comparative genomics (numan. rat and mouse) at the pronoter lewel. This tilows pradithon of ntw thangitipts sased on evidence fron multipie speriss as weth es devolopment of premizational ingetprints thet can sucessibly identily pronoters of sencs across speciss with $100 \%$ specincity such. pomparative pronoter andlysis is expesed to aid both tis arnoistion of new genomes via gene predictions and in the function-

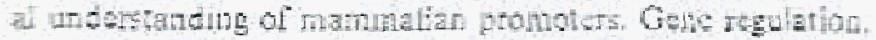

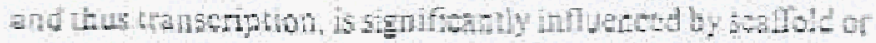
Fatrix shachment rogions (SMARs) as chscuscd by Juergon Bode (CBF Braunchwoh Gomany). SMARS art regions of DN, A that are always the first o towme single stranded typon tortiond stres an sureshblical DNA. SMARs can now be wisualted in situ, farilitating obarvation ot SMAR reiated cvents such as a tighter association with the ruslear nuatrix if genes wrehighly expresed. Tools heve but deweloped that will allow a mone detailed functional dissetwot of SMARs.

Lumoe Hust (Universty of Bath, Eagland) examined the physical distribution of esscntiat, and also wicely expressed. genes in yeast. Ho reported the preferential clustering of such gens independent of direst gene duphouton or coexprasion pattems. such maintenence of gene order is also supponted by the obscrution that cilustes of sscetial genes at least in you. rand to have reitively low rocombination rabe Sudhir Konat

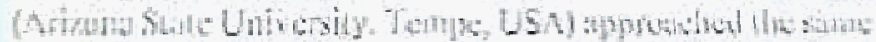
topit basw ou protein secuence couservation. Although the kind of molecular ciock that will be trost usetul for such andy-

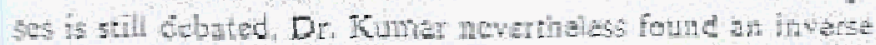
corrulation betwęen protein fenumee evolution and expreston level at least based on ESI databases, in genomes ragginfrom

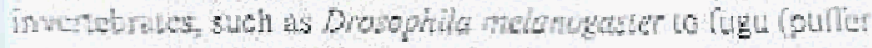

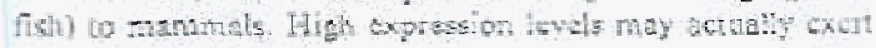

some selection prosture egainsh muthion Fixing in the comesponding Enes.

Regulat on of transcription by gene ongarizhtion bris boo: demonstraled in $C$ desuls, where uany genes wore rechily shonn by Com Etumentul (University of Colorado Heait: Scionces Cunter, Debvar, USA) to be orgmized into bacterial like optrons. Primay polycistronic Laseripls ate processot mite monots:ropic mRNA with trass-splicing into the downstrean whis of a wifom \$L2 leadu sequence. Mierasmays were pouder with the SL 3 soqutnce ideridying appoxintely 154 of $C$ degans gones that are expessed with approxhthate-

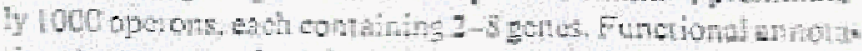

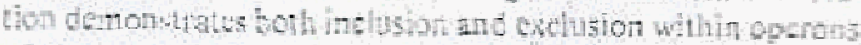

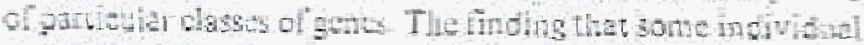
opcrurs sonthin funchionally rolated gants suggests that this featuc nay be use?til for prothing functions of wovel operthassocieted tones.

Danicl Fhicy (Fanard Medical School) dostibed reshlathon ol gene uxpression at the proteome level, spocitieally by the ubiquituan wa sysen. Largescale mass spectrosiopic analysis of ubiquitimited peptides from 5 . cerviside idumited $>70$ sub-

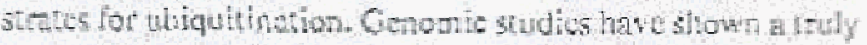
renizikable umber of potential ubictitination adapter pastcins, potentally twalmg kinsse gencs in number in mammals Exploration of Le roles of this system in repulating both tbuydancs and subcelluar locelization of specific proteins is onty int its begining stages.

Forces distating geneloction, shromosonut and rtanterip coud ratulion nsy well exicnd to rürovirgses that lodse in

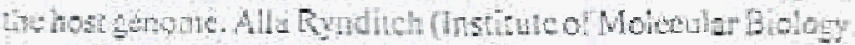

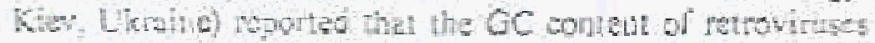
us ally nutches thit of the sochores of the hos genome where

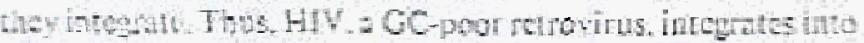
GG puor tagions of the how In contras, most cxpressed rtirgviruses ate louared in GC-fich rogions betouse these are preferontially the open and active parts of the chromatin. HIV appers to shange is preftred chromosonel onvionfatwat dur-

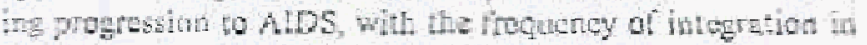

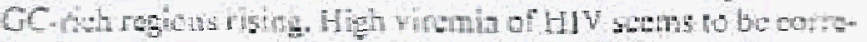
lated with isopyenic integration. HW also oxploits nueleo-cytopiantic frafieting to ture its sxpression, as mepoted by Rutt Brach-Wenen (GSF Research Center. Nutich, Gernany). Us-

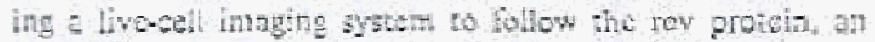
cisentiat postiransciptional regutator of HIV expression, it wes defermithd hat the tmport and export signals of this prothin are hrimstely conpled composite eluments and canitot bo clesing separa ed. It was found that this eveat is differenty reguisted in Heln cells and astrocyes. Therefore, cven al bust

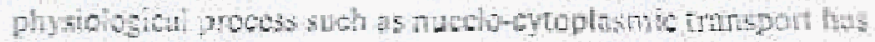

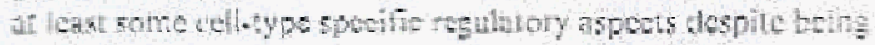
active in cwits set.

\section{RNA processing}

Jing Hue Yang (Yale Laversity] proviously desribed tha fenathably high levels of atenosine Lonversion to inosine in

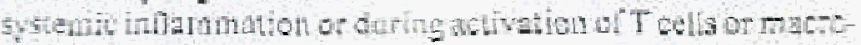


phages in vitro. New studies indicate that the level of eonvorsion can bi narkedly elevaled by owcrmpression of ADAR: and by adding lepromycin io inhibit necteat export of the deaninating croyne, suggesting that deamination may be an

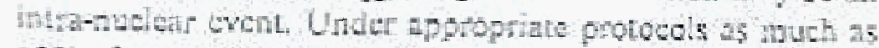
32 of aderosine can be eonverted to inazine. This sugests that gene cxpression, and penags evon tho nature of proteing product. may be merkedly alared in inflanmat tons secondary to posturanscriptional modifhatons of the mReA, The was Preiss (Viotor Chang Gudive Research Inatituie, Australis) descrbed how transmption and aIRVA tumover detcrmithe the quantitaive compostion of the cellutat transcrip-

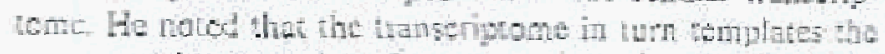

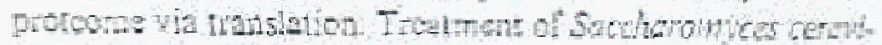

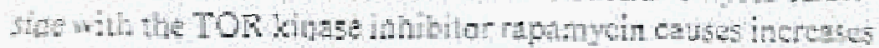
and docrages in the mRNA Levels of hugd:ads otgenes. In this

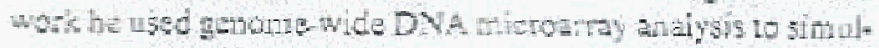
tancousy monitor traseriplome sud Iranslational changes for

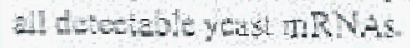

\section{Krocksuts for gene functional analysis}

Michad Boutros (Harvard Madisa) 5chool) diseusect a

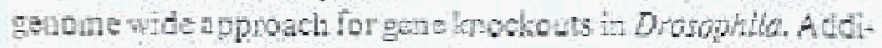
hion oi RWA gave bighk penerant and reproducible phenotyphs making it possible to undertake a high woughput sereaning of gene knuckoure basad on eall phanotypes, Assys have becn developed based on fuowscent of luminescent reporters and adapted to plate readeis as well as to phenotypo that can be derected by autometic mitrosogy

Nangy Hopkng and Adam Amsterdam (Massowusents Institue ou Tachnology, Cumbrdge, USA) dogcribed their largs" scale insertiotid! nulagusis project in zetortish, na which

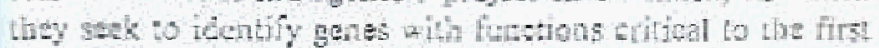
fredays of dewelopment They csimate that they have iduti.

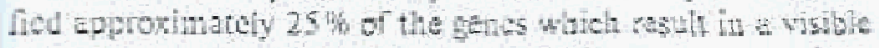
phenowge when mutated. Of these, $30 \%$ produce 5 mighe und seceific phenotype. To clarecterize the mutations. $70 \%$ of asceristad genes lave ben cloned. In acult fish, sereming For cence and early nomality idtutifed a numbel of cand. dele gones, amung then many cucoding conponents of che translational machinen, itcluding intrigungly, some tbo somal proteins.

\section{Gene-5pecific and disebse-specifio studies}

Profecia applying large and small sple proteomes ard network analyeg to suveral neurological diseoges and individue"

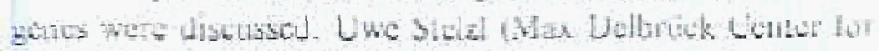

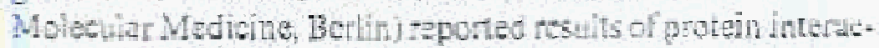
lien studies wh the Humington's Discase gane, HD. Using irgmens of HD and kiows interation pafmcts, a newoth comprisug 188 interactions tas cefraed. Artough poseibl: not all of the 167 movo interations are biologitaly televant 22 Fert werised in puldowh and oveley cxperiments, and runfThonat asägnnenes were nade for 10 Cellular processes of vasi- cle tsalicking. gloskeletal orgunization and thanswiptibn regtiation are impliesed in the nework It a smaller smie ndwork anaiys Rathe ew Gandine (University of Denver) conpied the known inienstions of chromosomo Il chooded geneswith components wh the MAP kinast and be ceicinewin signdimg pathwass. These intcractions pnotict the peturbation of thes:

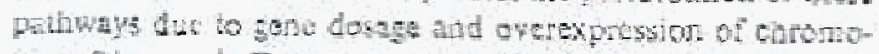
soune 21 genes in Down syndrome. Perturbarions in both pachways were demongrated th brins of the segnewal rtisomy

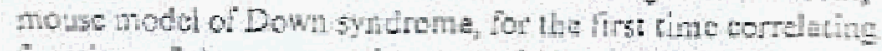
functions of chomosome 2 I gencs with a moleculat phonotype reigtwan to bevavior kind leaming

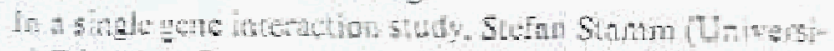
Ty es Erlangen, Grmany) deseibed aberran splielng of the Tau gere in lionowemporal ementia. Mutations hava boy

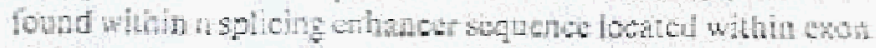
10 of the Tru gene. These mutations alce tnrerticion with the altemutivo splicing regulator, Trab and lead to abnormal pa:terns of cron 10 inelusion. Bento Sores (thiversity of lowa) cuscribed comparavive analysis of the pchomit stricturs of the meiastusis suppressor gene, Kiss1, in mouse. this genc shovs six altenntive splioe variants that encode thre dislinct proteins. In htman, the arthologs for wo of the thrue procing ene encodod lyy two separato but physiculy clasely reiacd. genes:

\section{Biginformaties}

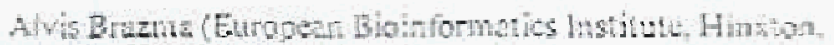
Eretatid) add:asod the elucidntion of the nework topwitgy

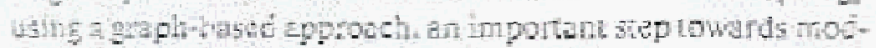
thitg and whithately undertanding colluha control systwms

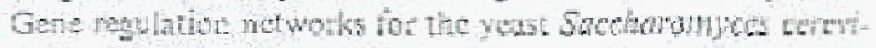
sive ware sompated, base on genomu wide dela sets from thes sincrent expariwenal techniques: chrometion immunt

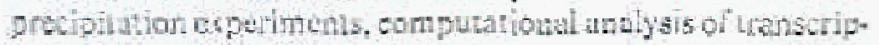

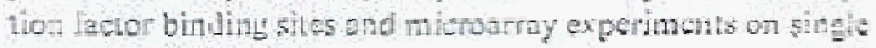
serc dutction nutunts.

Wins.on Hids South Afrism Mational Bioinfomaties In. stitute, Bellewils, South Aftica) addressed the problem of

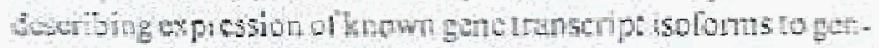

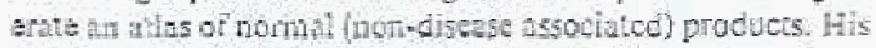

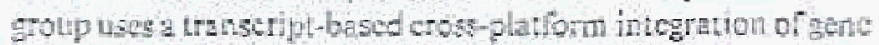
expebsion across ealtgotics of liuman anatomy. This woth has becn done in ollahoration with 3lologed Infomation: He-

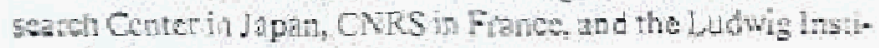
we Ro- Canoct Resedral in Braci.

Grazlato Puscle (University of Milaro, Italv) addrosed the identificat ion f conservat saqunce tup (CSTs) though com-

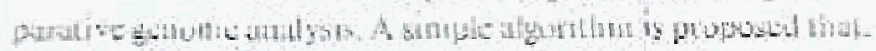

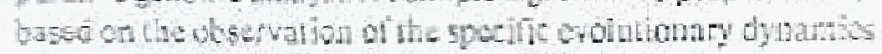

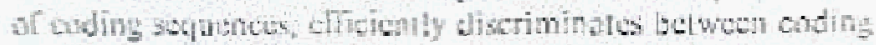
and hon cosm CST. The applusenos at this method muy halp the validation ul predieted gerses, the prodiction of alternative

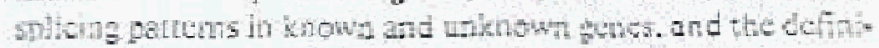
thon uf a diecionary of woh woding regulatory elements 
Martin Ving on (Man Planck Insitute for Molecular GenetWs and Computational Molcudar Biology. Berlis, Genamy) iatroduced cortepondence andysis as a tool for visualizing ascotiations bepwen genes and conditions in DNA-mikrosmay dria. The same tobluigue was algs apolied to emublish ascointions beween gent expression datd and iranserictior foctor binding sittes. While ton ycest this can be dont basud on published mansoription factor binding data, for butnan dath comparative analysis with nouse dsta in a search for bituding siles has bean used.

Jane: Thornton (Furapean Bipinformalics inslinte. Herts. tan, Ertglend) deseribed calculation of the ptysiochemical propartus of 745 mabolites from E. wh, based on their twe duchsiond stuctures obuired from the ExoCve and KEOG

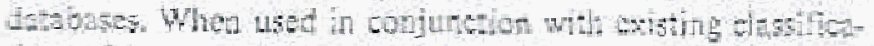
thons of he proteone, the bincligeperemess and promicuity of proteins and their ogenate fubstraces con bs elucidated

\section{Pesources}

Berghard Korn (R2PD, Heidelberg, Gemany) and Utw Radelof (RZPD, 1Bcrin, Gutranyl Caseffocd the rangeor produew and services available from the German Genomics Ru. source Conter. Now resounes include g growing sei of shuthe vectors and RWAi knowk down sonstructs. Osamu Ohara (Ka-

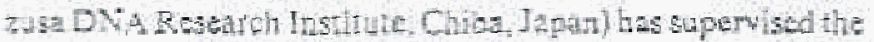
ceveloptrent and nidysic of the Klat hum and mouse cDNA sets, because of the apparco: relevelice of theso lone

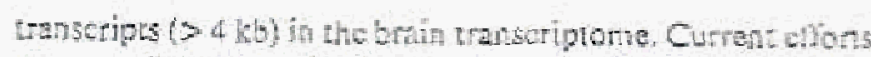
sire now directed to the functions: and $y$ sis of these gence. to this crd, the cDNA spquances are being cloned into expression vectors and polyclonal antibodies are beitug prodtuced. Dobres Cahti (Royal Colicge of Sunuons, Dublin, Iroland) thas conMructed a human brain cDNA cxpression libraty lor produsthot of a protein arry Curently more than 11,000 different protein equeness herwe buen werified. Prelininary streats whth antibodies to well charesteriged penes have revealed nown tross ruacil iles that sugect lack of shict specilicitios.

Geof Hicks (Uhiversity of Manitoon, Wistapes Canasa)

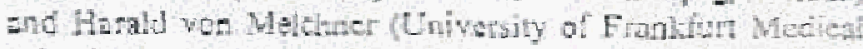
Sthogh, Gemany) deseribec the progits of the laterationd

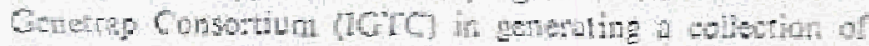

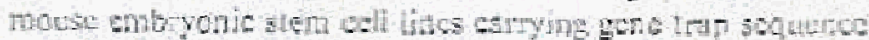
tags fCIST) from insertonal muragenesis. Ust of dittormt vectors with dilfernt mutationd mechenisms bas resultod in thoustnds of ES cell lincs wch with a distupt ion in a spurite knumn, genc. fell lines are described in a vintabse bint ane avalabte to mwestigators for construction of lenockart inice. In a resource of relaled ntility, Marin Hrabe de Angelis (GSF Institute of Experimenhal Genetics. Neukerberg, Geruatur) desribed the Crennan Mouse Chnic where nore thus 3000 mice put year can be pheyotyped for node that 160 paramblets.

Martin Ragmeld (The Jacson Iabaratory, Meine USA.

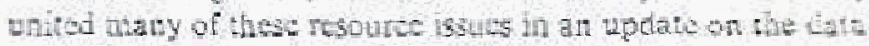
and serrch twols evallabla in the Mouse Expression. Datobasta which continuss with its eollection and integrariod oc all fortus of cxprosion date revana to the haboratoly nouse.

\section{Participants :}

\author{
tsabel Alusa \\ Uiniges: $-1 \mathrm{MMC}$ \\ Ruad so Campo alogre 823 \\ Ferte 450 - 180 Por \\ Telephone: +3512260749 月2 \\ Pax: +2517260915

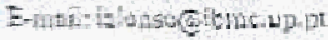

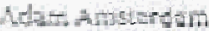 \\ Yaso-natis Iastibute of Tetholog: \\ Conter for Cunce Rusatih \\ 77 Mastolunetc Arense, E17-356 \\ Cambiag MAO2IJE IASA \\ Tcisphone +1-617-153-1605 \\ $\mathbb{P}=x+1-617+250.0258$

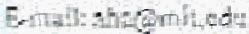 \\ Mintaur Anting \\ Inivessty of Edivorgh.

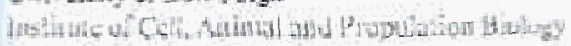 \\ King; Buldings Campus \\ E Hulvog EH, 9 I LK \\ Torphote +4a 01:31650 7405 \\ $F x^{2}+45(b)$ ij) $=4709$

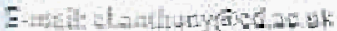

Har Alchiould

Resiul Instlutio

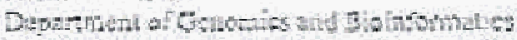

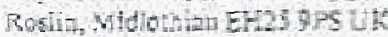

Telphotw +4401215274200

Par -4010131 400434

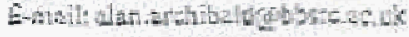

Iennitg ksung:

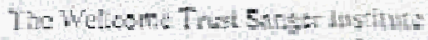

The Welleow: Trus Genour Cantus

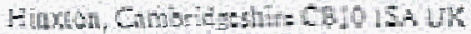

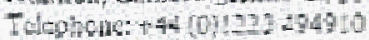

Fin: 444011223494515

B-mbil:jh: Quthanzech

Ton Bturtichthal

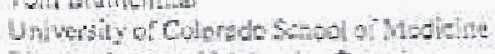

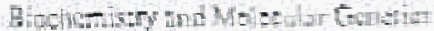

Diparangl,

4200 Enger 59 ateri us

Derrer, CO bozOL UEA

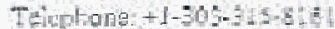

Fars, $+-30,3-315-6115$

E-inail comblumernalguehsudio
Jusment Bo:

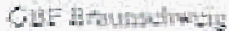

Epigenci: Rogutulou Depurwent

Masionods Wh 1

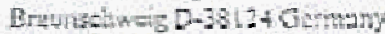

Telophone-\$5 coj:31 6151251

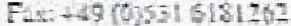

Email thogerfo:

Wicitander Datihy

Whivesity of hiats

Genem: Divirsty Centrs

Mvur Eante:

Ha iff 341057 trad

Thephune-4972 + 824h .48?

Fan'-47? $=8240362$

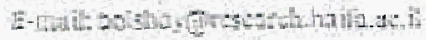

Higention llown

Sultant Mutinet Sthow:

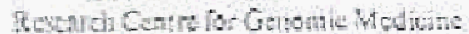

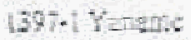

Hidak 75.-1241 Jophat

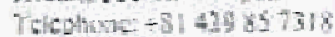

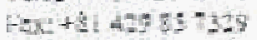

E-monil borogstintremed ac.jp 
Michat Boutras

Hartat Vedich Soliool

Deparmant of Gencties

200 Longwest Avesut

Boston, MA OZIIS USA

Talephone: $+1-617-432-7543$

Fox $\div-6 ! 7-432-7588$

E-mail mogeounavarded.

R-th Bractewsider

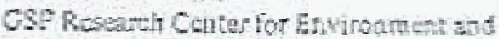
Heath

Inst:tute or Moloular Viroloty

loponedirr Lindstrose.

Musth D-85754 Germeny

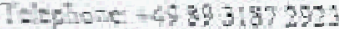

Fax: -45975187329

Evalitatatastdo

\section{Alvis Brazn:}

Europter Bioinformaltes Jnsthte

The W clicona Trus: Genome Canpus:

Huhabo, Crmbdeshire CEID ISO LK

Tilcphone 446 (0) $1223+494638$

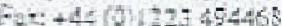

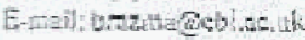

Maris Cascaitio

The Welcome Trus Swinger Ineitue

The Welente Trues Garaine Campes

Fhitiolot, Cumbridgeshife CB10 15. UK

Tolsphone -44 (0)1235494752

Fx: +44 (0)172) 494519

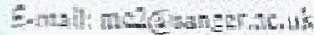

Prores Gutht

Centre Ier Heman Ptotoonics

Royal Collegu of Sungeons in Ireturt

125. 3. Sin 7 hat's Groon

Duthin 2 frelane

Telobone $+355 \mid-328550$

Fax +35314022453

E-mati enporsing

Give Elan:

UKHCMPHC

The whigone trus: Genome Canpe

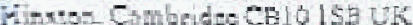

Tesphone +44 roy 1733 494563

Futh $+4 \div$ (0)1223 49.512

E-maili ge garehgmp arication

Suen Ftirlcy

Ghagu Lniversily

Cerpovine Satenco

17 Lilyourt Gartens

Glason CI2 S0O UK

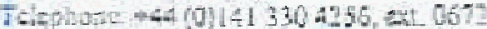

$E_{25}+44$ (0)1 $41250<915$

E-mati:aletes ghasuk

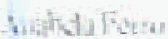

Wrigus - IMviC

Rue do Cserpo Alagk8 823

Potg a 190180 Potumat

Toteghous 451226079942

Pot +35:220092:5?

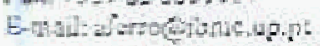

Destel Fing

Hutward Medies! Sehool

Department of Ceil biologi

240 Lomgood Angrut

Bomon. MA OLIJUSA

Twephen $-1-67+432+3422$

Fax: $\div|-6|-432-4 \mid$ the

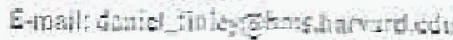

Drfen Fiors:

c.o

Grupo STAB

Sub Vids A Rerviblic

Detra 2781-60! Perrus:

Telephone: +3413 | 245046

Far: $-55121+4691+1$

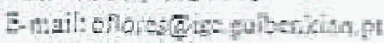

Tom Frengs:

MRE HCMPNC

The Welleothe T rus Gesems

HinkLu, Cmbritgohire CDIO ISD UR

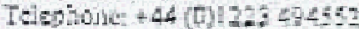

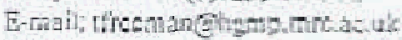

Twane Furac

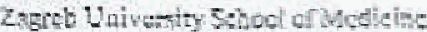

Deparment of Furenge Moditite

and Gintinclesy

Salat 11

Zagreb 10000 Crattia

Ttephone: + 735 t 4592250

Fax: +38514500235

ב-nali: ivanaromet lie

Kathetem Garcing

Haingreity or Dews:

7895 Guve:s sted:

Dether, 0030205 LS

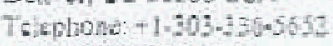

$73 x+-107.252847$

Engil kgriseguts st.

Tom Gingeres

Anymentios ine

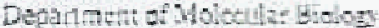

39ou Cuntral Esoneswoy

Sunt Cand CA $95051 \mathrm{LSA}$

Tulaghon: $\div 1-405-0731-5048$

$F_{4 x-1}-408-451.0423$

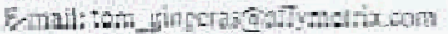

Phitis Goadwh

Qiegen LWA

Danquilc Survices

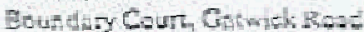

Crawles RHIO SIAX UK

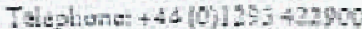

F⿻x: +44 (0) $25 \div 42529$

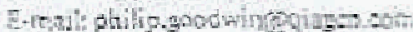

Willtulat

Uhimaty s: Oleseno

Bianformalics Rosutit Centectompun

Scivice

Lnyority Avenue

Giutow GI2 800 LK

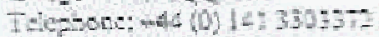

F $2 x-44$ (0) $413: 02627$

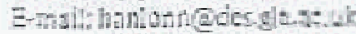

Anr Hothey

Uniwesity or Edis burgh

ICA:

Kunguilding

Edinbute EFS JIM UK

Teicolone atik (0)131 6507403

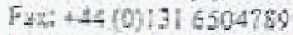

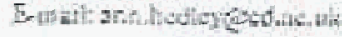

John Heskoth

Lilivesity ar Namengte

School or Cet and Malecutar Biortenes

Kings Roa

Nercasth-upen-Tyne NEI 7RU LK

Jeientand +44 10191 $2384+4$

Fin: +4A (0)

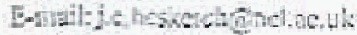

GenTHids

Mathidw lasitule of Cell Bigloge and

the Univerely ar Mantroba

Dhtrimgal of Biochenistry uted

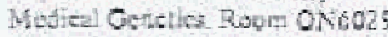

G75 MidDerne? AweTh

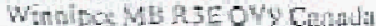

Telephas: $+204-787-2135$

Fix: $+1-204-787=2190$

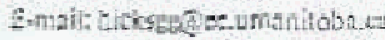

Wingen Fide

SANE:

University Wedum Cvpt

Mesederda: Roud

Bolwithe 7505 South Aftico

Telephoak $\times 27219393544$

Fan +2721959 1512

E-ust: minituesunbiacan

Naror Hopkins

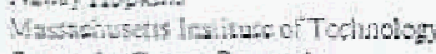

Cover tor C-ceer Reversh

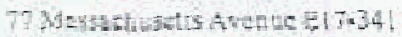

Combrilge, MA 02U52 USA

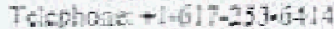

Five +1-617.255-22.38

E-tinail nhophingauitade

Martine Hras de Angelis

QSt GmbH

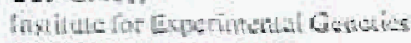

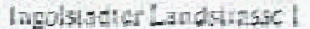

Newhotge Des:s4 Germany

Tolephose - 498931872302

Fax:-4 39 31875300

Embats hrotestid:

Leureace thure

Litivutefily or Bath

Doporumen of ololgy and Bodmisty

BHA BA27AY UK

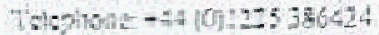

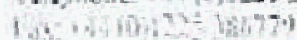

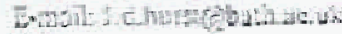


Keuric Jebosch

Tha Wutrone Jrust Sengor linsitute

The Welkase Thus Geromo Cumpos

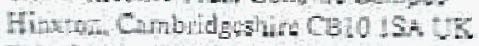

Triphowe +44 (0)1725 49497)

Fac - $44(0) 1372494912$

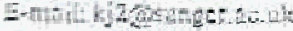

Daniele Remater

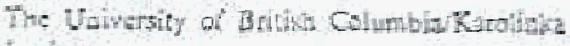
Irostint:

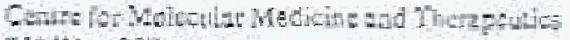
TSO Wert 283 A werius

Yansourer W32 $4 \mathrm{H} d \mathrm{C}$ Cands

TAluphas $-1-604-855 \cdot 2342$ tex. 7947

Fan-1-100+-675-3s:

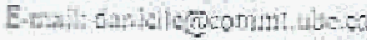

Cespokoangs

Wolisan i-stiture or Bionted bal Resents

Gumbestot

Londey WCIE 6AU UKK

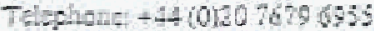

Fax $=4402079165954$

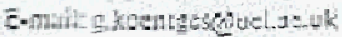

Bembiard kioth

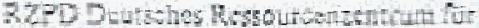

Covomiench uig Gmb:

ThF 5 so

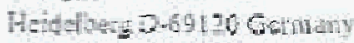

Telphate +45.6221 424700

$F_{3 x-49621424704}$

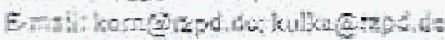

Suchir Suma

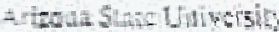

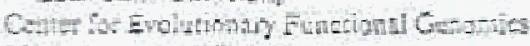

Lifterience A Wing

Tarpo, A2 aE287-1501 USA

Terotore $+1.400-727.5949$

$=2 x-1-450-105-2514$

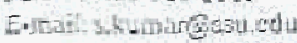

Riglaterist:

CS.Motcento formen Grom

und Detelopmen!

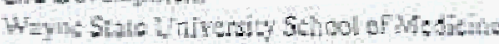

Ousterses and Gywologt

275 E Hencock

Dwail, M! 49201 USA

Tolopdat + $+-313-7+5-760 \mathrm{~g}$

$5 x-1.312-75+7073$

Emait riedehgnetwaynedu

Rull Lereitg

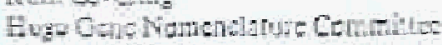

Farke Hbusen Eesmon Rond

Cuaring Fisain, Kont TN27 0.LX LK

Tolabiowe -44001233713441

B-mail: rub Givalen weinc. Lh.

Witk Lascornbe

Cthire fo- Suruesura Biolegy

Departutent el Molcudar Biphystes and

Biokimisto. BSS 437

Yale Laiverity. P.O Bos. 208174

NEW Haven, CT OSSD-B!1 WSA.

Telmous $-1-201-4325405$

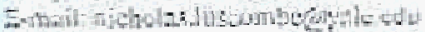

Ghistian Mrerbing

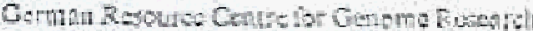

Gent Exprustor Proflitise

Im Keuthoimer Fold so

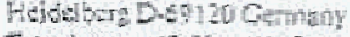

Tolephone: 4962214244

Fox:-412 6221 434704

E-mait em-tekerethide

Davicharin

Livivasty ar Dunde

Pos-Genante d Mclesuht hateractions Gentre

Dow suset

Duntion DDI AED Sertan

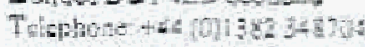

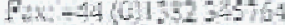

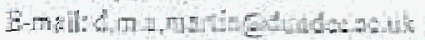

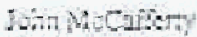

The Wellecme Tnat Senge Atatho te

The Wolitome Trus: Genowa Gimpus

Hinutr, Cambrideshisu CBIO LSA JTh

Telopiche -4A (0)12t3 49492:

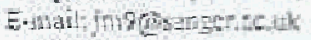

Aneribshospoh

Whivering of Tary

Departmert of Bhyestadote-

Rilizast

Teriu 51010 Estong

Telephena: +372737502 s

Fax: +372742028

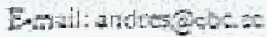

Sergiv Nos

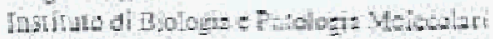

Uniwhith La Sipignte

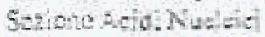

Burcolo A kom 5

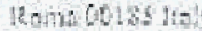

Tolecions:-3906 499:22412h

frx: -3906491200

Emuli gergonsigusteme in

Gestinu Ohaus

KuZuS DVA Restere: listitule

Departwent or Human Grae Residerth

2-G-7 KazUSa-kemalati

Kyำกz:

Chibu 29200518 Jupen

Tetephond $+81+38523913$

Fuxi $+81+38523912$

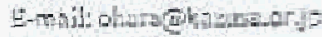

Graximo Porsic

Univesit of Mitat

Phelology and Goneri bionenistry

Departmicat

viscostate

Milan $20: \$ 3$ italy

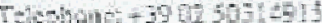

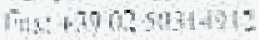

Fonail arzamopolem mintit it
Thowas Prets:

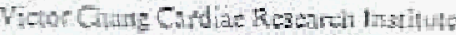
Meledur Gentes Programe

384 Vienvis 5 tros

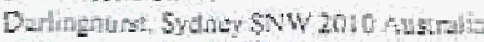
Tdephones +5124903332

Ext -61293959501

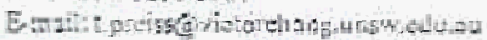

Johes wollat

Gomen Cefert Resureh Center

Division of Funetional Oenone Analysis

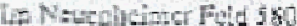

Hoideberg D.GS 120 Gemia.

7 wphy +49622141468 t

$F_{2 x}+-052,47468 ?$

E-mitipullzadods

Ele Pundito- Hurn

Uinivumity o: Oiesgery

Cemputing Scionso

tivberk Gardens है-17?

Cissow 01? 800 UK

Telophoc $=-4(0) 1413305024$

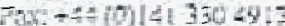

E-paid dadesglasuk

Utw Ristor

RPDG GmbH

Produstion Damulnent

Haubnerwas 6

Belin D-145s9 Crmany

Teluphses $+4930326312 ?$

Fax +493012639127

E-natl: realareredide

Auns Rinkter?

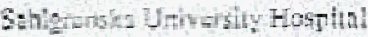

povertment of Laboralory Moltenst.

Cliniel Chereistr.

Gistebure $3+1145$ Swatten

relogune $-46,3 ; 3422374$

Fax: $-40.31829+43$

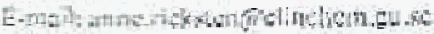

Marise Ring-eld

The fochon Laburstory

$60 p \mathrm{Mu}$ Sinct

Tur Hirtber. ME 04509 USA

Ticphone +1-207-286-6458

Fex:- $-127.185-5132$

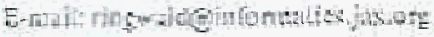

Piefer Rolitim

Gheri Univonits

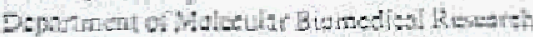

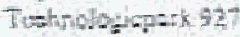

Ghent (7witaurde) B-905 B Baim

Teicolwac + 32,5315600

Fax $+329313609 / 13500$

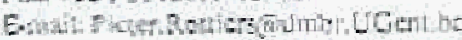

Mita Ryoding

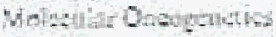

150 Zabcianoge Sirg:

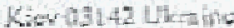

Teluphone +32042653498

Fix $+980<4600759$

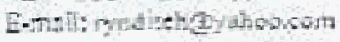




\author{
Mavelisentor \\ STAB VIDA \\ Buptiment of Gảnomis \\ Ar Resulitia \\ Oarr $7781-64$ I Partual \\ Telephon:-5il 12:4469652 \\ Fax +251 121440942

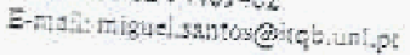

\section{Rezsibuis}

Lomserity of ctinhurg:

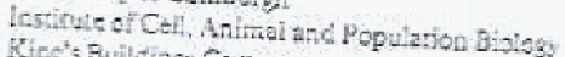

Rirets Bulloing Campus

Edinunel SHS SIT LK

Toenon: $+44(0) 131650743$

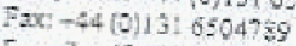

5-rati molfobiadowok

\section{Mratad Snyder}

Yale Uniwsiby

D-pacment or Molctular, Celinlar and

Developmotri siology

New H2man, CT 06520-8109 LISA.

thalmutenc: +1-205-432-6139

$F x+1 \cdot 20+32-397$

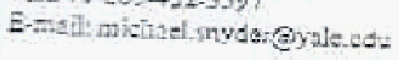

Beuro Sourbs

Univergity ofllows

Roy I and Iuflle A Garw

Collaw-orsedione

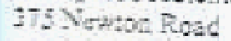

4184 Modiud Eduation

Brometici" Resescit Fadily

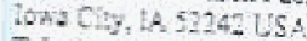

Tdaphere $-1-311-355.5250$

Fax: $-1-319-395-9965$

Ertual:mento-searesgulowt odu

forinspled:

Wahington Univasiy

Comom Sonuencine Conter

44E4 Forwa Park-Cunpus Bor 5501

S: Lour, MO $5: 10$ USA

Tolephohts -1-3/4-786-1800

Fat-1-314236-1810

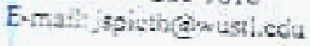

Stefen S-und

Uniwerify bo Ertengen

Institus to Bughenias

Fabrutase 17

Erlaten D-\$1084 Gomany

Tolephone -29913 15. 34622

Fax-45915 552460

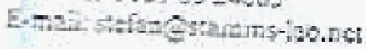

Eugenes 5erdov

institute of Molesular Genwirs

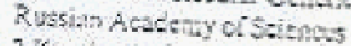

2 Kurutaray $5 \mathrm{~g}$.

Mowow 123162 Rens:

Terephone +7095 iswous

Fut +7055196031

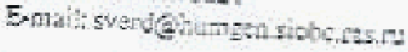

Marku Tenplin

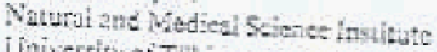

Jubersity a: Tubinge

Beparmsn, of Microarny Trshnolocy

Markwewnats.

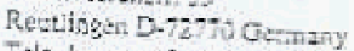

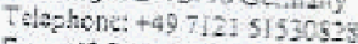

Fan +497121 5151016

E-nail tomplingumide

Giare Thompso:

Quiget Ite.

Genonies Serwices

Boundary Cour, Catwich Ros:

Crwher RLI $1094, \mathrm{UK}$

Telephasa -44 (b)1295 -22000

Th: 54 (o) 1253422912

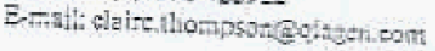

Dine Thothing

EMBDites

Welcone Trust Gonome Campes

Himion, Canbridgabirc CD:G ISD UK

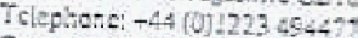

$F 2 x_{0}+4 \div(0) 1227494456$

E-rakthortiongebine tox

Mithedels Torkd

Gracind Bfology

Middlewar Hous

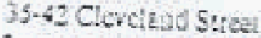

Land ty W1T 4LB. UK

Telchow: +44 (0)20 7631 255

$79 x+440207631906]$

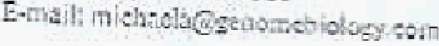

tyuld unyla Tsyot

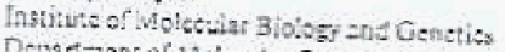

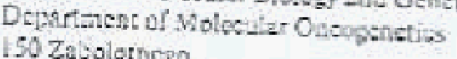

150 Zabolering a

Kuv 03 ! 43 Ukmine

Telphone $+3 \mathrm{BN} 4=265343 \mathrm{~B}$

Fax +580442653495

E-mailkasangimbospus

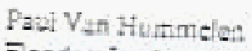

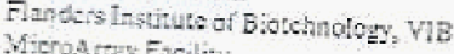

teroatru Facility

Herestrat 4?

Latren 500 Befgivan

Tolehorts: -52163479.39

Prux:-32:60:75:40

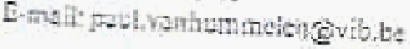

Martin Wueres:

Mex-Plane insinut fer Molochar Ceneriss

Deparment orbiodalcrmaties

Theursers

9milin D.7ergs Gentany

Tdehowe $+49(0)=084: 3 ! 15 !$

Fox: 499019084131152

E-mide vingurgholgon.mpg.dt

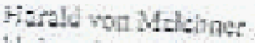

Unitwaity of Frakfunt Medred scheol

Laboratiog for Molceular Hemetolots

Thesde-Sirm-Kai ?

Frankfur D-6059o Strinumy

Telephone 49 \&5 63016096

Fhx:-\$9 69 6301 650

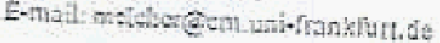

Shemsa Whismen

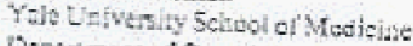

Bepartment or Gencties

295 Conston Avenus - BCMM 336

Nerw lovan, Cl essio tish

Ictophonc +1-205-7572a

Fax $+1207-737-2186$

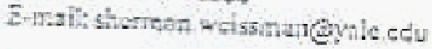

7homs: wemer

Gononath 5ofruge GmbH

Landshersw Sir it

Munit D-6os3 Germy

Titichothen: +4585 5997660

Fux 40 a9 5997555

E-mail Hinn gemomulx ds

Fibghua Yong

Hele Untursity School or Medicine

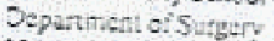

331 Cedar Sinel

Now Fiven, CI 06:20 USA

Teltphone +4.201-737-5595

Fax $-1-205-37$-5954

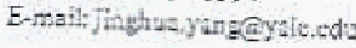

